\title{
Dynamics of Bulk Viscous String Cosmological Model in $f(R, G)$ Theory of Gravity
}

\author{
Kailas R. Borgade ${ }^{1 *}$, S. R. Bhoyar ${ }^{2}$, Vineeta Basotia ${ }^{3}$ \\ ${ }^{I}$ Research Scholar, Shri Jagdishprasad Jhabarmal Tibrewala University, Jhunjhunu, India \\ ${ }^{2}$ Department of Mathematics, Phulsing Naik College, Yavatmal, India \\ ${ }^{3}$ Department of Mathematics, Shri Jagdishprasad Jhabarmal Tibrewala University, Jhunjhunu, India
}

\begin{abstract}
Existing investigations dedicated to the self-propelling investigation of dynamics of bulk viscous string in LRS Bianchi type-I cosmological model within the circumstance of alternative theory of gravity with Langrangian be the impulsive perform of Ricci scalar $R$ and Gouss-Bonnet invariant $G$, say $f(R, G)$ gravity. Exact solutions of the field equations correspond to special law of variation which provides singular model. Also some physical and kinematical aspects and its behavior with the present day universe of the cosmological model have been discussed.
\end{abstract}

Keywords: Bulk Viscous String, Bianchi Type-I space-time, $f(R, G)$ Gravity.

\section{Introduction}

Modified gravity is one among the foremost promising candidates for explaining the present fast enlargement of the Universe, and even its unification with the inflationary epoch. Yet, the big selection of models capable of explaining the phenomena of dark energy (DE) imposes that current analysis focuses on an additional precise study of the doable effects of changed gravity on each cosmological and native levels. Recent data-based information on the cosmic growth history has cause the invention of accelerated growth of the universe. It's believed that the rationale for this is often associated in nursing exotic style of unknown force with large negative pressure dubbed as DE. However, the character and behavior of DE remains a mystery. There are two major approaches to handle this drawback of cosmic acceleration either by introducing a DE element within the Universe and study its dynamics or by decoding it as a failure of Einstein's general theory of relativity and think about modifying Einstein's theory of gravitation termed as "modified gravity approach".

Among the assorted modifications of Einstein's theory $f(R), f(R, T), f(G), f(R, G)$ theories of gravity etc. etc. that provides a natural unification of early-time inflation and latetime acceleration are attracting a lot of and a lot of attention. Bulk viscosity or volumetrically viscosity introduces damping related to volumetrically straining. Its purpose is to boost the modeling of high-speed dynamic events. String cosmological models square measure vital throughout structure formation within the early stages of evolution of the universe.
Spontaneous symmetry breaking through the phase change within the early universe lead to a random network of stable line like topological defects acquainted as cosmic strings. It's typically notable that huge strings functioned place to begin for the big structures like cluster of galaxies within the universe. Therefore, these string models have gained wide attention of Researchers. Many authors within the literature have investigated vital aspects of string cosmological models in varied theories of gravitation. Many authors and researchers studied the dynamics of bulk viscous string cosmological models of the universe.

Pradhan [1] examined some circularly symmetric inhomogeneous thick liquid string cosmological models with attractive field and cosmological term $\Lambda$ differing with time. Czajka et al. [2] considered mass consistency in a warm QCD model with enormous number of tones at two limit restricts; the powerless and the very strong't Hooft couplings. Bishi and Mahanta [3] researched the Bianchi type-V string cosmological model with mass consistency in $f(R, T)$ hypothesis of gravity by thinking about an uncommon structure and straightly fluctuating deceleration boundary. Singh [4] considered anisotropic Bianchi-V Universe with attractive field and mass thick liquid in string cosmology. Gusu [5] introduced Bianchi Type-I universe within the sight of mass thick and DE liquid nature of a cosmological model. Hegazy [6] proposed the structure of the gravitational hypothesis dependent on Lyra math and the overall hypothesis of relativity, he examined Bianchi type I cosmological model within the sight of gooey liquid. Çağlar and Aygün [7] examined mass thick with bizarre quark matter joined to the string cloud for higher dimensional Friedman-Robertson-Walker (FRW) universe in Lyra calculation. Adhav et al. [8] acquired Kaluza-Klein cosmological arrangements in n-measurements for quark matter combined with the string cloud and space dividers overall relativity. Sharif and Waheed [9] investigated locally rotationally symmetric Bianchi I universe in Brans-Dicke gravity with self-interfacing potential by utilizing charged gooey cosmological string liquid. Singh and Ram [10] examined the elements of spatially homogeneous and anisotropic Bianchi type-III string cosmological model in presence of mass thick liquid and electromagnetic field. Singh et al. [11] investigated with absolutely anisotropic Bianchi type- 
II cosmological models loaded up with a mass thick liquid within the sight of time-differing gravitational and cosmological constants. Borkar and Ashtankar [12] introduced an answer of LRS Bianchi type-II space-time with string thick liquid and attractive field by tackling the Barber's field conditions of self-creation hypothesis of attraction. Santhi et al. [13] determined $f(R)$ gravity field conditions with the assistance of a spatially homogeneous and anisotropic Bianchi type-III space-time, within the sight of a mass thick liquid, containing one-dimensional enormous strings. Katore et al. [14] considered a LRS Bianchi type-I space time to investigate mass gooey string model in altered $f(G)$ gravity. Hatkar and Katore [15] researched mass thickness combined with inestimable string for Bianchi type VI0 cosmological model with regards to Self-creation hypothesis of attractive energy. Smash et al. [16] discovered new spatially homogeneous Bianchi-I cosmological model of the universe loaded up with a mass thick liquid in gravity hypothesis. Smash and Kumari [17] introduced nonparticular Bianchi types I and V cosmological models, within the sight of mass gooey liquid and inside the structure of $f(R, T)$ gravity hypothesis. Vidyasagar et al. [18] determined Brans-Dicke (Phys. Fire up. 124, 925 (1961)) field conditions with the assistance of a spatially homogeneous and anisotropic Bianchi type-VI0 metric, within the sight of a mass gooey liquid, containing one-dimensional enormous strings. Velagapudi and Davuluri [19] introduced spatially homogeneous Bianchi types II, VIII, and IX string cosmological models with mass consistency in Nordtvedt's (Astrophys. J. 161:1059, 1970) general scalar-tensor hypothesis of attraction with the assistance of a unique case proposed by Schwinger. They saw that solitary the mass thick cosmological model exists on account of Bianchi type IX universe. Prasanthi and Aditya [20] contemplated a spatially homogeneous and anisotropic Kantowski-Sachs space-time loaded up with mass gooey liquid, containing one-dimensional grandiose strings. Rao and Neelima [21] concentrated pivotally symmetric string cosmological models with mass consistency in Brans-Dicke (1961) and general relativity (GR). Allot et al. [22] introduced a mass thick charged Bianchi type IX string cosmological model in everyday relativity.

As, there are so numerous change of activity in GR are gotten, such $f(R), f(R, T), f(G), f(R, G)$ etc. and so on These sensibly extraordinary fascination hypotheses are an undertaking to build a semi-traditional subject inside which GR and the vast majority of its independent alternatives are frequently recuperated. An undemanding and a standout amongst other alteration to general relativity that the $f(R, G)$ hypothesis of gravity, which is the capacity of Ricci-scalar $(R)$ and Gauss-Bonnet gravity $(G)$. Till now a few models of $f(R, G)$ hypothesis are arranged.

Cruz-Dombriz and Saez-Gomez [23] zeroed in on the examination of an sort $f(R, G)$ altered gravity, the supposed gravity, and they play out a profound investigation on the solidness of significant cosmological arrangements. Elizalde et al. [24] considered DE cosmology in an extremely changed Gauss-Bonnet model of gravity with and keeping in mind that not a field. Laurentis et al. [25] examined cosmological swelling in the system of gravity, where $f$ is a conventional capacity of the curve scalar $R$ and the Gauss-Bonnet topological invariant $G$. Shekh et al. [26] dedicated to the dynamical investigation of Relativistic Hydrodynamics with some thermo dynamical qualities in $f(R, G)$ gravity towards spatially homogeneous isotropic cosmological model loaded up with isotropic liquid.

\section{Metric, $f(R, G)$ Gravity and Dynamics Bulk Viscous String}

We consider a LRS Bianchi type-I space-time of the form

$d s^{2}=d t^{2}-A^{2} d x^{2}-B^{2}\left(d y^{2}+d z^{2}\right)$,

Where, $A$ and $B$ are the potential functions of cosmic time $t$ only.

So many authors [5], [6], [14] contemplated the above said model because of its physical importance that it's solid and anisotropic, from which the method of isotropization of the universe is studied through the passage of time conjointly play a significant role in understanding and outline of the first stages evolution of the universe wherever because the theoretical argument and therefore the trendy experimental information from CBR radiation and therefore the LSS observations, support the existence of anisotropic phase that turns into isotropic one. Therefore, the model devouring anisotropic background that approaches to property at late times is a lot of correct for the outline of entire evolution of the universe.

The most general action for $f(R, G)$ is given as:

$S=\frac{1}{2 k} \int d^{4} x \sqrt{-g}\left\{R+f(G)+S_{M}\left(g^{j k}, \phi\right)\right\}$,

Where, $S_{M}\left(g^{j k}, \phi\right)$ is the matter action, $R$ is the Ricci Scalar and $G$ is the Gauss-Bonnet invariant defined by:

$G=R^{2}-4 R_{r s} R^{r s}+R_{r s \alpha \beta} R^{r s \alpha \beta}$,

where the notations $R_{r s}$ and $R_{r s \alpha \beta}$ are occupied for the Ricci and Riemann tensor respectively.

Variety of standard activity (2) regarding metric (1) gives the accompanying gravitational field condition:

$$
\begin{aligned}
& R_{\alpha \beta}-\frac{1}{2} g_{\alpha \beta} R=k T_{\alpha \beta}^{m a t}+\sum_{\alpha \beta} \\
& \text { Where, } \sum_{\alpha \beta}=\left[\begin{array}{l}
\nabla_{\alpha} \nabla_{\beta} f_{R}-g_{\alpha \beta} \Xi f_{R}+2 R \nabla_{\alpha} \nabla_{\beta} f_{G} \\
-2 g_{\alpha \beta} \Xi f f_{G}-4 R_{\alpha}^{\lambda} \nabla_{\lambda} \nabla_{\beta} f_{G} \\
-4 R_{\beta}^{\lambda} \nabla_{\lambda} \nabla_{\alpha} f_{G}+\Xi_{\alpha \beta} f_{G}+4 g_{\alpha \beta} R^{\alpha \beta} \nabla_{\alpha} \nabla_{\beta} f_{G} \\
+4 g_{\alpha \beta} R^{r s} \nabla_{r} \nabla_{s} f_{G} \cdot 4 R_{\alpha r s} \nabla^{r} \nabla^{s} f_{G} \\
-\frac{1}{2} g_{\alpha \beta}\left(f_{R} R+f_{G} G-f(R, G)\right) \\
+\left(1-f_{R}\right)\left(R_{\alpha \beta}-\frac{1}{2} g_{\alpha \beta} R\right)
\end{array}\right]
\end{aligned}
$$


Here, $\nabla_{\alpha}$ represents the covariant derivative.

$f_{R} \equiv \frac{\partial}{\partial R} f(R, G)$ and $f_{G} \equiv \frac{\partial}{\partial G} f(R, G)$ gives the partial derivatives of $f(R, G)$ with respect to $R$ and $G$ respectively.

In this work we obtain the solution of field equations and the behavior of the universe using some kinematical and physical quantities towards the $f(R, G)$ gravity model of the form:

$f(R, G)=f_{0} R^{n-1} G^{-n} ; f_{0}>0$ be constant. For the values of constant $n$, two types of gravity models are recovered:

I)

$f(R)$ gravity model corresponding to $f_{0}=1, n=0$ and

II) $\quad f(G)$ gravity model corresponding to $f_{0}=1, n=1$

Odintsov et al. [27] studied certain classes of $f(R, G)$ gravity which have engaging phenomenological highlights, concerning the effective acknowledgment of the DE and of the inflationary period. Especially, they examined the overall formalism and showed how a few inflationary and DE advancements can be depicted with regards to $f(R, G)$ gravity.

S. H. Shekh [28] introduced examination committed to the dynamical examination of anisotropic DE LRS Bianchi type-I cosmological model with regards to changed gravity in which Langrangian be the discretionary capacity of Ricci scalar and Gouss-Bonnet invariant say $f(R, G)$ gravity in the way of anisotropic fluid.

For the fluid string with bulk viscous fluid, the energy momentum tensor is taken as,

$T_{j k}=\rho u_{j} u_{k}-\lambda x_{j} x_{k}-\xi \theta\left(g_{j k}+u_{j} u_{k}\right)$,

Here $\lambda=\rho-\rho_{p}$ is the string tension density, $\rho$ is the energy density, $\rho_{p}$ being the particle energy density, $\xi$ is the bulk viscosity of the fluid, $u^{j}$ is the four velocity vector of particles and $x^{j}$ is the unit space like vector which gives the direction of the string given by,

$u^{j}=(0,0,0,1)$ And $x^{j}=\left(A^{-1}, 0,0,0\right)$ such that $u_{j} u^{j}=1=x_{j} x^{k}$ and $u_{j} x^{k}=0$ so from (2.5) we get,

$T_{j}^{k}=[-\lambda+\xi \theta,-\lambda+\xi \theta,-\lambda+\xi \theta, \rho+\lambda]$

Sahoo et al. [29] researched Bianchi-III and VI0 cosmological models with string liquid source in $f(R, T)$ gravity with regards to late time speeding up extension of the universe as proposed by the current perceptions. Mishra and Dua [30] gave another class of mass thick string cosmological model in Saez-Ballester hypothesis of attraction. Katore and Shaikh [31] examined cosmological model with peculiar quark matter connected to the string cloud overall hypothesis of attraction for pivotally symmetric space time. They got model with the assistance of unique law of variety for Hubble boundary proposed by Bermann (Nuovo Cimento B 74:182,
1983). Bhoyar et al. [32] analyzed Kantowaski-Sachs cosmological model with mass gooey and grandiose string in the structure of Teleparallel Gravity supposed $f(T)$ gravity, where $T$ denotes the torsion scalar. Singh and Baro [33] researched Bianchi Type-I string cosmological model with mass thick liquid and negative consistent deceleration boundary in everyday relativity.

\section{Field Equations and Solutions}

In the extant of bulk viscous string source given in equation (6), the field equations (4) corresponding to the metric (1) lead to the following set of linearly independent differential equations of the form:

$\left\{\begin{array}{l}{\left[\frac{\dot{A}}{A}+2 \frac{\dot{B}}{B}\right] \dot{f}_{R}-12 \frac{\dot{A}}{A} \frac{\dot{B}^{2}}{B^{2}} \dot{f}_{G}+\frac{1}{2}\left(R f_{R}+G f_{G}-f\right)} \\ +\left[2 \frac{\dot{A}}{A} \frac{\dot{B}}{B}+\frac{\dot{B}^{2}}{B^{2}}\right] f_{R}\end{array}\right\}=\rho+\lambda$

$\left\{\begin{array}{l}-\ddot{f}_{R}-2 \frac{\ddot{B}}{B} \dot{f}_{R}+8 \frac{\dot{B}}{B} \frac{\ddot{B}}{B} \dot{f}_{G}+4 \frac{\dot{B}^{2}}{B^{2}} \ddot{f}_{G}-\frac{1}{2}\left(R f_{R}+G f_{G}-f\right) \\ +\left[2 \frac{\ddot{B}}{B}+\frac{\dot{B}^{2}}{B^{2}}\right] f_{R}\end{array}\right\}=-\lambda+\xi \theta$

$\left\{\begin{array}{l}-\ddot{f}_{R}-\left[\frac{\dot{A}}{A}+\frac{\dot{B}}{B}\right] \dot{f}_{R}+4\left[\frac{\dot{B}}{B} \frac{\ddot{B}}{B}+\frac{\dot{B}}{B} \frac{\ddot{A}}{A}\right] \dot{f}_{G}+4 \frac{\dot{A}}{A} \frac{\dot{B}}{B} \ddot{f}_{G} \\ -\frac{1}{2}\left(R f_{R}+G f_{G}-f\right)+\left[\frac{\ddot{A}}{A}+\frac{\ddot{B}}{B}+\frac{\dot{A}}{A} \frac{\dot{B}}{B}\right] f_{R}\end{array}\right\}=-\lambda+\xi \theta$

In equation (7), if we use $\lambda=\rho-\rho_{p}$ then the resulting equation for particle density is:

$\left\{\begin{array}{l}{\left[-\frac{\dot{A}}{A}-2 \frac{\dot{B}}{B}\right] \dot{f}_{R}+\left[12 \frac{\dot{A}}{A} \frac{\dot{B}}{B^{2}}\right] \dot{f}_{G}-\frac{1}{2}\left(R f_{R}+G f_{G}-f\right)} \\ -\left[2 \frac{\dot{A}}{A} \frac{\dot{B}}{B}+\frac{\dot{B}^{2}}{B^{2}}\right] f_{R}\end{array}\right\}=\rho_{p}$

Using the values of $\rho$ and $\lambda$ in (7) and then putting it in (8) the equation we get,

$[2(\gamma-1)-1]\}\left\{\begin{array}{l}{\left[\frac{\dot{A}}{A}+2 \frac{\dot{B}}{B}\right] \dot{f}_{R}-\left[12 \frac{\dot{A}}{A} \frac{\dot{B}}{B^{2}}\right] \dot{f}_{G}+\frac{1}{2}\left(R f_{R}+G f_{G}-f\right)} \\ +\left[2 \frac{\dot{A}}{A} \frac{\dot{B}}{B}+\frac{\dot{B}^{2}}{B^{2}}\right] f_{R}\end{array}\right\}=p$

Dividing both sides of (11) by $(\gamma-1)$ we get,

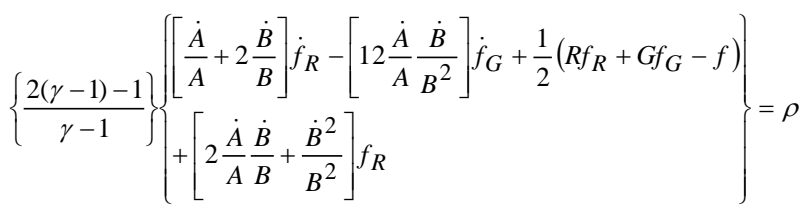


By subtracting (12) with (10) and using $\lambda=\rho-\rho_{p}$ we get,

$$
\left\{\left(\frac{2(\gamma-1)-1}{\gamma-1}\right)+1\right\}\left\{\begin{array}{l}
{\left[\frac{\dot{A}}{A}+2 \frac{\dot{B}}{B}\right] \dot{f}_{R}-\left[12 \frac{\dot{A}}{A} \frac{\dot{B}}{B^{2}}\right] \dot{f}_{G}} \\
+\frac{1}{2}\left(R f_{R}+G f_{G}-f\right)+\left[2 \frac{\dot{A}}{A} \frac{\dot{B}}{B}+\frac{\dot{B}^{2}}{B^{2}}\right] f_{R}
\end{array}\right\}=\lambda
$$

Now by using (13) in (7) we get,

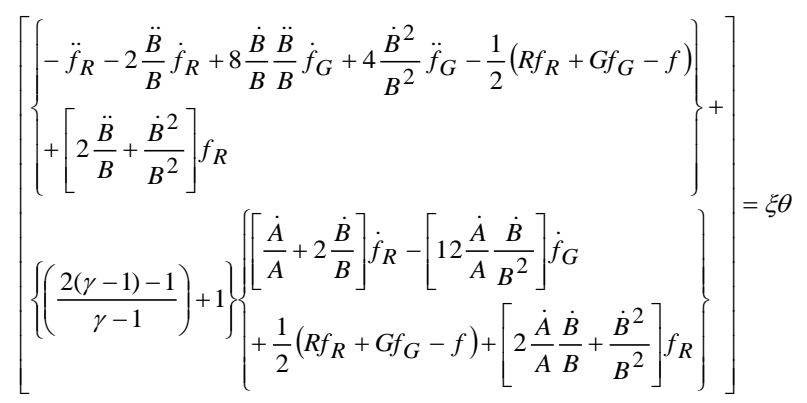

The spatial volume and average scale factor severally outline as,

$$
\begin{aligned}
& V=\sqrt{-g}=A B^{2} \\
& a=V^{1 / 3} \text { so, } a=\left(A B^{2}\right)^{1 / 3}
\end{aligned}
$$

In 1983 Berman proposed the unique law of variety of Hubble's boundary which yields a consistent worth of deceleration boundary of the universe, as indicated by Berman's law,

$$
q=\frac{d}{d t}\left(\frac{1}{H}\right)-1
$$

The generalized mean Hubble parameter that expresses the enlargement rate of the reference frame, is given as,

$$
H=\frac{\dot{a}}{a}
$$

Where, a is the average scale factor which is given by the equation,

$$
a=\left(e^{\eta t}-1\right)^{1 / \eta}
$$

As the creator [31] talked about the technique for extraordinary law of variety and by taking inspiration from this we additionally utilize this strategy to discover the upsides of our infinite possible capacities.

Conduct of scale factor of an elements of mass thick string cosmological model versus time with the adequate choice of constants as demonstrated inside the accompanying Fig. 1. While noticing the graphical idea of the scale factor which is the capacity of time; it has been presumed that the scale factor is increments dramatically with limitless time span.

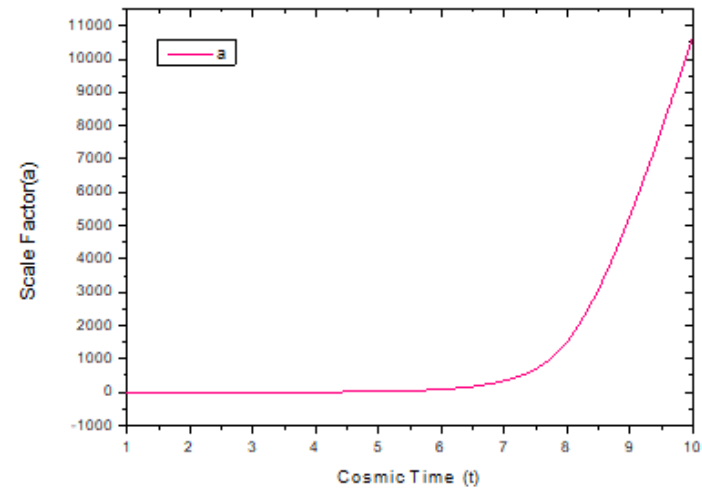

Fig. 1. Behavior of scale factor of dynamics of bulk viscous string cosmological model versus cosmic time with the appropriate choice of constants $\eta=0.2$.

From equations (16) and (19), the metric potential comes out to be and we take $A=B^{m} ; m \neq 0$ with this we get the values of $\mathrm{A}$ and $\mathrm{B}$ as follows:

$B=\left(e^{\eta t}-1\right) \frac{3}{\eta(m+2)}$
$A=\left(e^{\eta t}-1\right) \frac{3 m}{\eta(m+2)}$

With the help of (20) and (21) spatially homogeneous and anisotropic locally rotationally symmetric Bianchi type-I cosmological model with dynamics of bulk viscous string source within the framework of $f(R, G)$ theory of gravity becomes:

$d s^{2}=d t^{2}-\left[\left(e^{\eta t}-1\right) \frac{3 m}{\eta(m+2)}\right]^{2} d x^{2}-\left[\left(e^{\eta t}-1\right) \frac{3}{\eta(m+2)}\right]^{2}\left(d y^{2}+d z^{2}\right)$

In the model (22), it is seen that both metric possibilities are the remarkable and having power term. Here we saw that both metric possibilities are again the elements of infinite time t. Consequently, at first the model has no peculiarity except for at the point, $\mathrm{t}=0$, it address a particular model and everywhere time i.e. $t \rightarrow \infty, A, B \rightarrow \infty$.

\section{Physical Properties of the Model}

The Ricci Scalar and Gauss-Bonnet invariant $G$ with the values of metric potentials (20) and (21) for the model (1) is written in the following way:

$R=-2\left[\left\{\frac{9 m^{2}-3 \eta(m+2)^{2}+18 m+27}{(m+2)^{2}}\right\} \frac{e^{2 \eta t}}{\left(e^{\eta t}-1\right)^{2}}+\left\{\frac{\eta(3 m+6)}{(m+2)}\right\} \frac{e^{\eta t}}{\left(e^{\eta t}-1\right)}\right]$

and

$G=216\left[\left\{\frac{3 m^{2}+6 m \eta-m \eta(m+2)(2 \eta+1)}{(m+2)^{4}}\right\} \frac{e^{4 \eta t}}{\left(e^{\eta t}-1\right)^{4}}+\left\{\frac{3 \eta m}{(m+2)^{3}}\right\} \frac{e^{3 \eta t}}{\left(e^{\eta t}-1\right)^{3}}\right]$

As we probably are aware, the Riemann tensor could be a useful asset to control whether the peculiarity is vital or amass. 
In the event that the curve develop into boundless at a specific reason, the peculiarity is significant. We can develop totally various scalars from the Riemann tensor thus it very well may be affirmed whether they develop to be endless some place or not. A boundless scope of scalars will be place along from the Riemann tensor, in any case, balance reflections will be acclimated show that there square measure just a limited scope of self-deciding scalars.

Calculated Energy density of the particle by using the potential functions (20) and (21) in (10) is as:
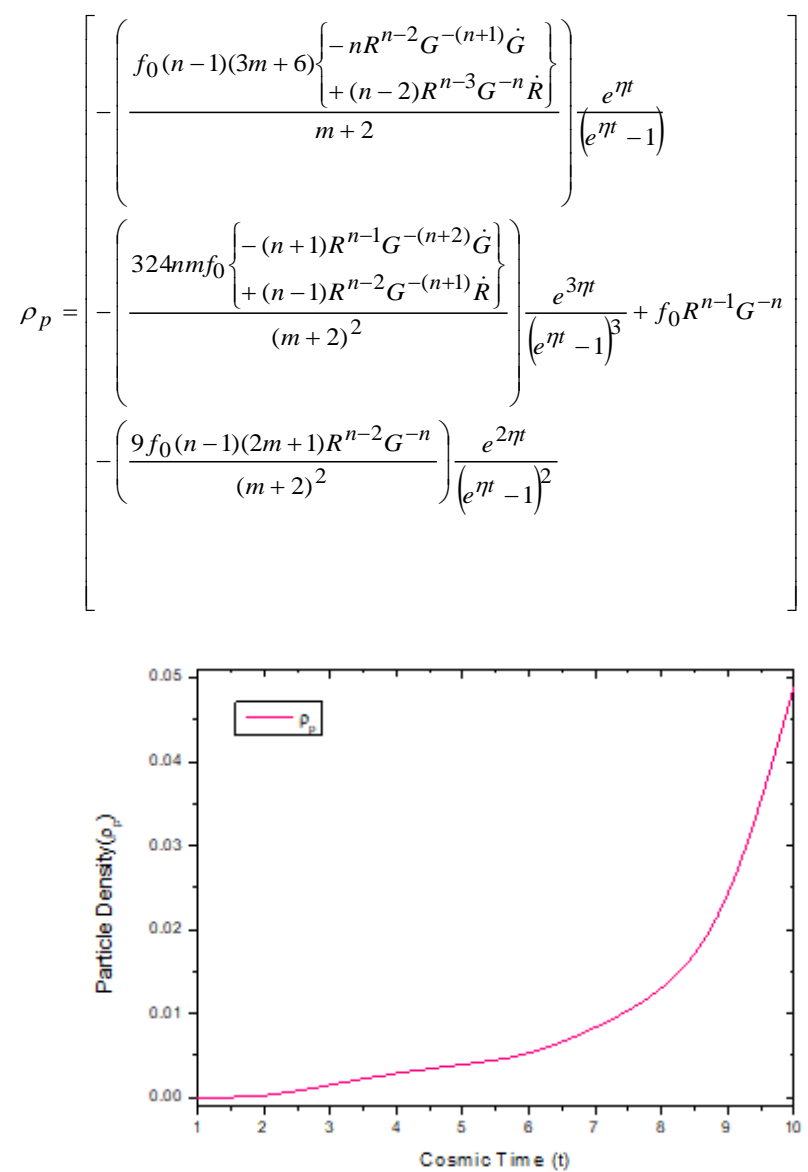

Fig. 2. Behavior of particle density of dynamics of bulk viscous string cosmological model versus cosmic time with the appropriate choice of constants $m=2, n=3$ and $\eta=0.2$.

The molecule thickness (24) and the energy (26) thickness of a mass gooey string evaporates at $\mathrm{t}=0$ and increments vastly at boundless inestimable time span. This shows qualities of scale factor and consequently we reason that the two densities are positive type capacity of astronomical time t. The graphical idea of molecule thickness is appeared in Fig. 2 and the graphical conduct of energy thickness of gooey string cosmological model is appeared in Fig. 4.

Calculated pressure of the universe by using the potential functions (20) and (21) in (11) is as:

$$
p=\{(\gamma-1)-1\}\left[\begin{array}{l}
\left.\left(\begin{array}{l}
f_{0}(n-1)(3 m+6)\left\{\begin{array}{l}
-n R^{n-2} G^{-(n+1)} \dot{G} \\
+(n-2) R^{n-3} G^{-n} \dot{R}
\end{array}\right. \\
m+2
\end{array}\right\}\right) \begin{array}{l}
e^{\eta t} \\
\left(\begin{array}{l}
e^{\eta t}-1
\end{array}\right)
\end{array} \\
\left(\begin{array}{l}
324 n m f_{0}\left\{\begin{array}{l}
-(n+1) R^{n-1} G^{-(n+2)} \dot{G} \\
+(n-1) R^{n-2} G^{-(n+1)} \dot{R}
\end{array}\right\} \\
(m+2)^{2}
\end{array}\right) \frac{e^{3 \eta t}}{\left(e^{\eta t}-1\right)^{3}}-f_{0} R^{n-1} G^{-n} \\
+\left(\frac{9 f_{0}(n-1)(2 m+1) R^{n-2} G^{-n}}{(m+2)^{2}}\right) \frac{e^{2 \eta t}}{\left(e^{\eta t}-1\right)^{2}}
\end{array}\right]
$$

Conduct of pressing factor of an elements of mass gooey string cosmological model versus time with the adequate choice of constants as demonstrated inside the accompanying Fig. 2. The generally speaking nature with three distinct models or the case for the model are appeared in the accompanying picture.

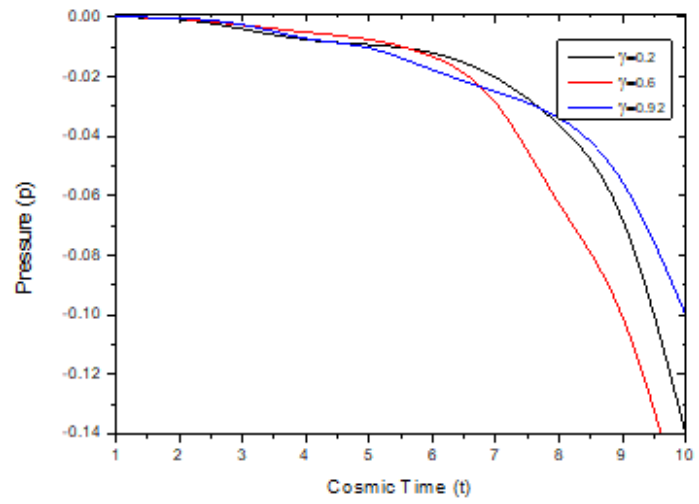

Fig. 3. Behavior of pressure of dynamics of bulk viscous string cosmological model versus cosmic time with the appropriate choice of constants $m=2, n=3$ and $\eta=0.2$.

Calculated Energy density of the universe by using the potential functions (20) and (21) in (12) is as:

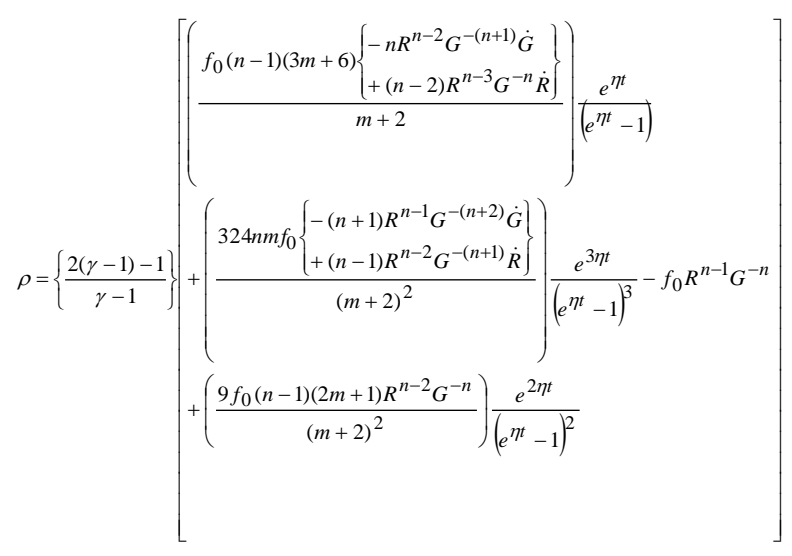

The behavior of energy density of a dynamics of bulk viscous string cosmological model versus time with the acceptable selection of constants as shown within the following Fig. 4. The overall nature with three different models or the case for the model are shown in the following picture. While observing it; 
it's clearly seen that for all three models of the universe the energy density is increases.

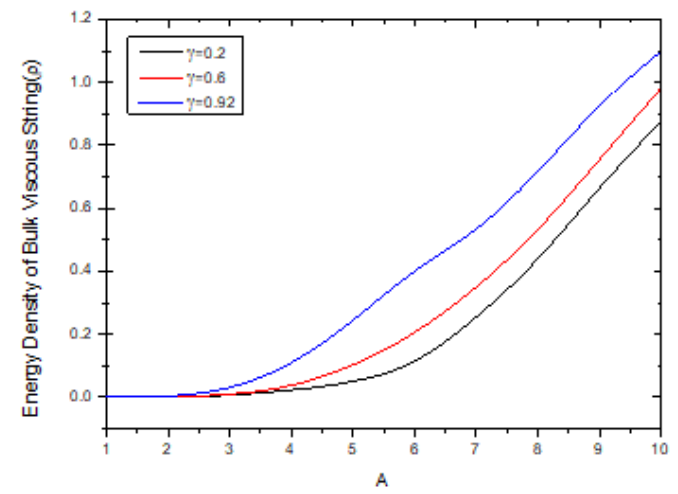

Fig. 4. Behavior of energy density of dynamics of bulk viscous string cosmological model versus cosmic time with the appropriate choice of constants $m=2, n=3$ and $\eta=0.2$.

Calculated tension density of the string universe by using the potential functions (20) and (21) in (13) is as:

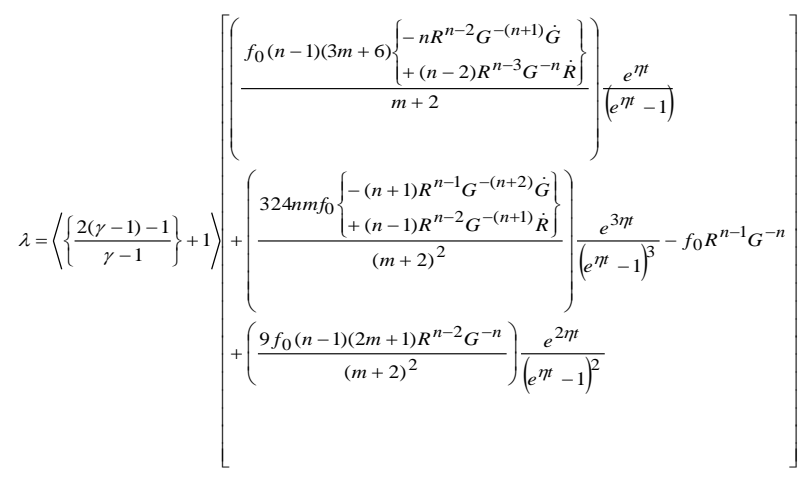

Behavior of string tension density of a dynamics of bulk viscous string cosmological model versus time with the acceptable selection of constants as shown within the following Fig. 5. The overall nature with three different models or the case for the model are shown in the following picture. While observing it; it's clearly seen that for all three models of the universe the tension density of bulk viscous string is increases.

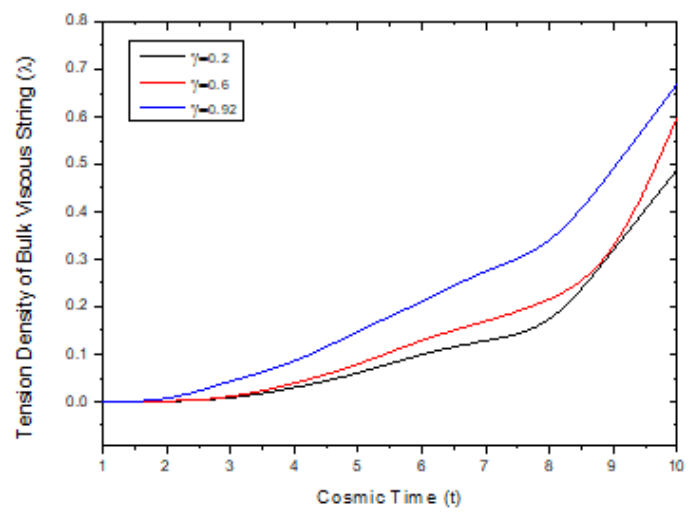

Fig. 5. Behavior of string tension density of dynamics of bulk viscous string cosmological model versus cosmic time with the appropriate choice of constants $m=2, n=3$ and $\eta=0.2$.
The obtained bulk viscosity equation after proper substitution of potential functions and the expansion scalar we got a bulk type of equation for it and is given as;

$$
\begin{aligned}
& {\left[-\left\langle\frac{f_{0}(n-1)}{3}\left\{\begin{array}{l}
-n\left\langle G^{-(n+1)} \ddot{G}+(n+1) G^{-(n+2)} \dot{G}^{2}\right\rangle R^{n-2} \\
-\left\langle 2 n(n-2) R^{n-3} G^{-(n+1)} \dot{G} \dot{R}\right\rangle \\
+(n-2)\left\langle R^{n-3} \ddot{R}+(n-3) R^{n-4} \dot{R}^{2}\right\rangle G^{-n}
\end{array}\right\}\right)\right\rangle \frac{\left(e^{\eta t}-1\right)}{e^{\eta t}}} \\
& -\left(\frac{2 \eta f_{0}(n-1)}{m+2}\right)\left\{\begin{array}{l}
-n R^{n-2} G^{-(n+2)} \dot{G} \\
+(n-2) R^{n-3} G^{-n} \dot{R}
\end{array}\right\}\left[\begin{array}{l}
\frac{3-\eta(m+2)}{\eta(m+2)} \frac{e^{\eta t}}{\left(e^{\eta t}-1\right)}+1
\end{array}\right]
\end{aligned}
$$

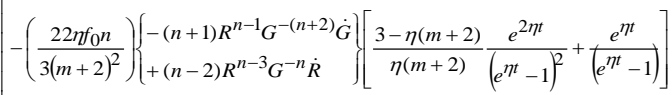

$$
\begin{aligned}
& -\left(\frac{12 f_{0} n}{(m+2)^{2}}\right)\left\{\begin{array}{l}
-(n+1)\left\langle G^{-(n+2)} \ddot{G}-(n+2) G^{-(n+3)} \dot{G}^{2}\right\rangle R^{n-2} \\
-\left\langle 2(n+1)(n-1) R^{n-2} G^{-(n+2)} \dot{G} \dot{R}\right\rangle \\
+(n-1)\left\langle R^{n-2} \ddot{R}+(n-2) R^{n-3} \dot{R}^{2}\right\rangle G^{-(n+1)}
\end{array}\right\} \frac{\left(e^{\eta t}-1\right)}{e^{\eta t}} \\
& \left.\xi=+\left(\frac{f_{0} R^{n-1} G^{-n}}{3}\right) \frac{\left(e^{\eta t}-1\right)}{e^{\eta t}}+\left(\frac{f_{0}(n-1)}{3}\right)\left\{\frac{27-6 \eta(m+2)}{(m+2)^{2}} \frac{e^{\eta t}}{e^{\eta t}-1}\right)+\frac{6 \eta}{m+2}\right\} R^{n-2} G^{-n} \\
& +\left[\begin{array}{l}
\left(\begin{array}{l}
f_{0}(n-1)(3 m+6)\left\{\begin{array}{l}
-n R^{n-2} G^{-(n+1)} \dot{G} \\
+(n-2) R^{n-3} G^{-n} \dot{R}
\end{array}\right\} \\
3(m+2)
\end{array}\right) \\
\left.\left(\frac{2(\gamma-1)-1}{\gamma-1}\right\}+1\right) \\
+\left(\begin{array}{l}
\frac{108 n m f_{0}\left\{\begin{array}{l}
-(n+1) R^{n-1} G^{-(n+2)} \dot{G} \\
+(n-1) R^{n-2} G^{-(n+1)} \dot{R}
\end{array}\right\}}{(m+2)^{2}} \\
\left(e^{\eta t}-1\right)^{2} \\
\left(\frac{f_{0} R^{n-1} G^{-n}}{3}\right) \frac{\left(e^{\eta t}-1\right)}{e^{\eta t}} \\
+\left(\frac{3 f_{0}(n-1)(2 m+1) R^{n-2} G^{-n}}{(m+2)^{2}}\right)
\end{array}\right) \frac{e^{\eta t}}{\left(e^{\eta t}-1\right)}
\end{array}\right]
\end{aligned}
$$

Bulk viscosity vanishes at initial time $t=0$ and it get increases with time. Hence with this we conclude that the bulk viscosity is the positive function with respect to the cosmic time. This shows that the universe is moving to singularity.

\section{Kinematical Properties of the Model}

The kinematical properties which are important in cosmology for discussing the geometrical behavior of the universe that are spatial volume, Hubble parameter, expansion scalar, mean parameterized isotropy parameter, shear scalar, deceleration parameter and overall density parameter which have the following expressions by using the potential functions (20) and (21) as follows:

The obtained spatial volume of the model is,

$$
V=\left(e^{\eta t}-1\right)^{3 / \eta}
$$




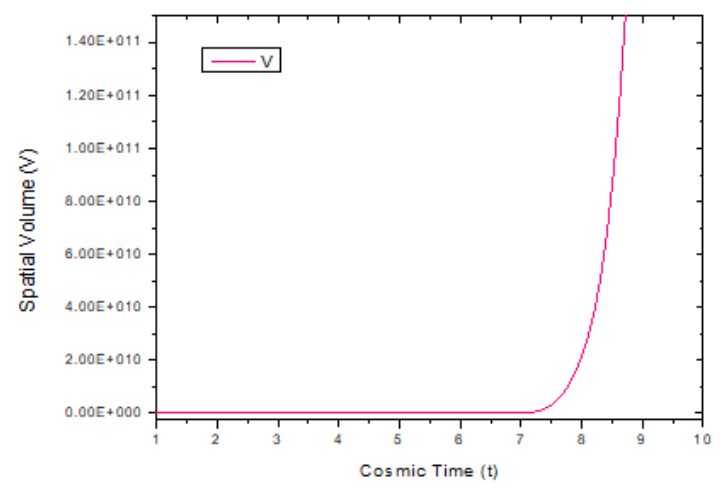

Fig. 6. Behavior of spatial volume of dynamics of bulk viscous string cosmological model versus cosmic time with the appropriate choice of constants $\eta=0.2$.

The resulting mean Hubble parameter of the model is,

$$
H=\frac{e^{\eta t}}{\left(e^{\eta t}-1\right)}
$$

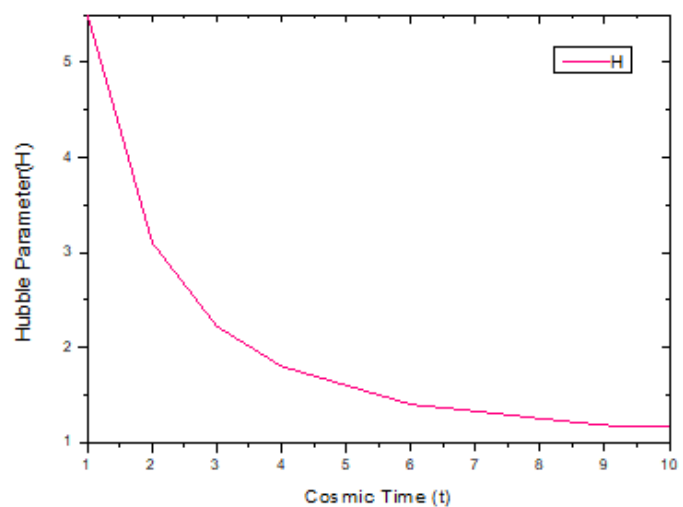

Fig. 7. Behavior of Hubble Parameter of dynamics of bulk viscous string cosmological model versus cosmic time with the appropriate choice of constants $\eta=0.2$.

The obtained expansion scalar for the model is,

$$
\theta=\frac{3 e^{\eta t}}{\left(e^{\eta t}-1\right)}
$$

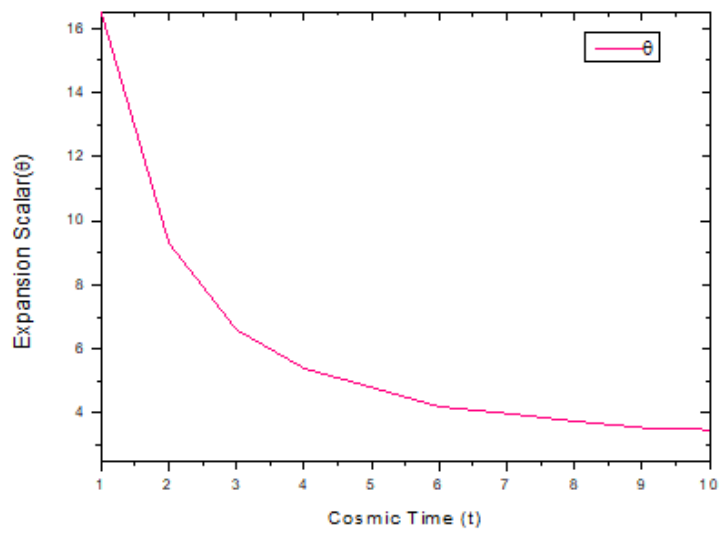

Fig. 8. Behavior of expansion scalar of dynamics of bulk viscous string cosmological model versus cosmic time with the appropriate choice of constants $\eta=0.2$.
Behavior of Hubble parameter and the expansion scalar of a dynamics of bulk viscous string cosmological model versus time with the acceptable selection of constants as shown within the following Fig. 7 and Fig. 8 While observing the equation (30) and (31) it is clear that both Hubble parameter and the expansion scalar are the reciprocal function of exponential terms and it is observed that it is decreasing with time that means at $t=0$ the Hubble parameter and expansion scale moving towards infinity. Hence this shows that the model gets expanding but with very small rate of expansion. The graphical nature shows in the Fig. 7 and Fig. 8.

To mentioned whether or not the models either approach isotropy or not, we tend to outline associate anisotropy parameter of the enlargement as, $A_{n}=\sum_{i=1}^{3}\left(\frac{H_{i}-H}{H}\right)^{2}$

$A_{m}=\frac{2(m-1)^{2}}{(m+2)^{2}} ; m \neq 1$

From equation of anisotropy it can be concluded that model of the universe shows anisotropy at $m \neq 1$ and showing isotropy at $m=1$ all through evolution.

Similarly the founded Shear Scalar of the model is $\sigma^{2}=\frac{3}{2} H^{2} A_{n}$

$$
\sigma^{2}=\frac{2(m-1)^{2}}{(m+2)^{2}} \frac{e^{2 \eta t}}{\left(e^{\eta t}-1\right)^{2}}
$$

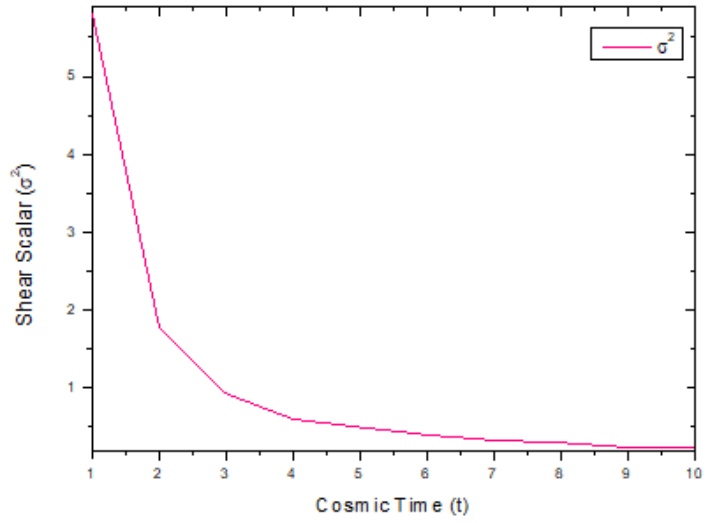

Fig. 9. Behavior of shear scalar of dynamics of bulk viscous string cosmological model versus cosmic time with the appropriate choice of constants $m=2$ and $\eta=0.2$.

The behavior of the shear scalar of bulk viscous string cosmological model with time for suitable choice of constants is as shown inside the subsequent Fig. 9. It is observable that the shear scalar of bulk viscous string is moving towards vanishing position at infinite time but at time $t=0$ it is infinity and hence its behavior can be observed in the following Fig. 9.

The obtained deceleration parameter of the model is given by, $q=\frac{\eta}{e^{\eta t}}-1$ 
The conduct of a deceleration boundary of elements of the mass gooey string a cosmological model versus a period with the reasonable selection of constants as demonstrated inside the resulting Fig.10. By choosing appropriate values of constants it is observed that the deceleration parameter is the negative function of cosmic time $t$. At $t=0, q=-0.8$; which shows that the universe has an accelerated expansion. It is documented that once $\mathrm{q}$ is negative then the model universe expands with acceleration, and once $q$ is positive then it explains a contracting universe. Though these observations like cosmic microwave background radiation and SNe-Ia advised the negative value of $\mathrm{q}$ however it is often remarkably state that they're not capable to deny regarding the decelerating expansion of universe.

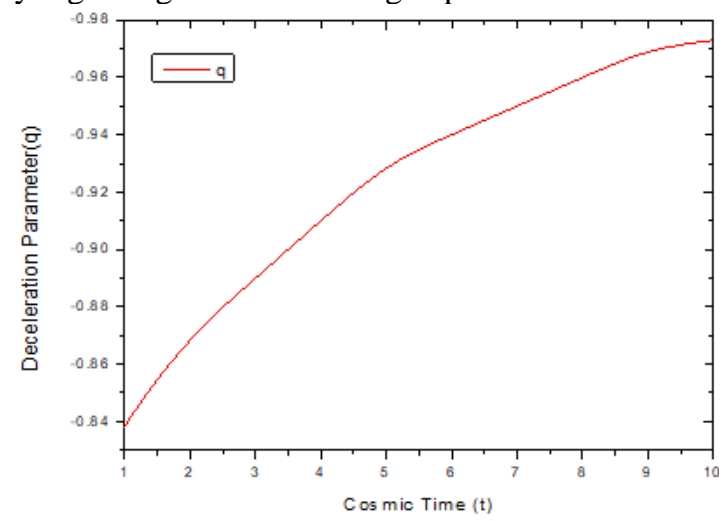

Fig. 10. Behavior of deceleration parameter of dynamics of bulk viscous string cosmological model versus cosmic time with the appropriate choice of constants $\eta=0.2$

In the cosmology the jerk parameter can be defined as the third derivative of a scale factor and is written as:

$j(t)=\frac{\dddot{a}}{a H^{3}}=\frac{e^{\eta t}-\eta}{e^{\eta t}}+\frac{e^{2 \eta t}-\eta^{2}}{e^{2 \eta t}}-\frac{\eta^{2} t\left(e^{\eta t}-1\right)}{e^{2 \eta t}}$

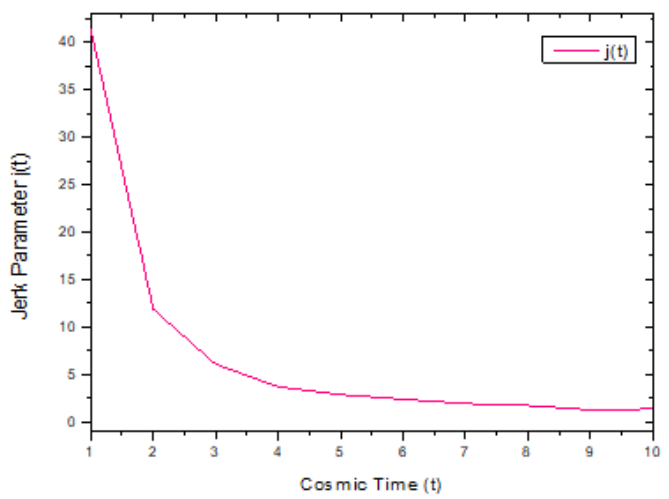

Fig. 11. Behavior of jerk parameter of dynamics of bulk viscous string cosmological model versus cosmic time with the appropriate choice of constants $\eta=0.2$.

The jerk parameter shows the regular bounce of the universe and the jerk parameter is decreasing function with time and get infinity at $t=0$.

The calculated overall density parameter of the model of universe is, $\Omega=\frac{\rho}{3 H^{2}}$

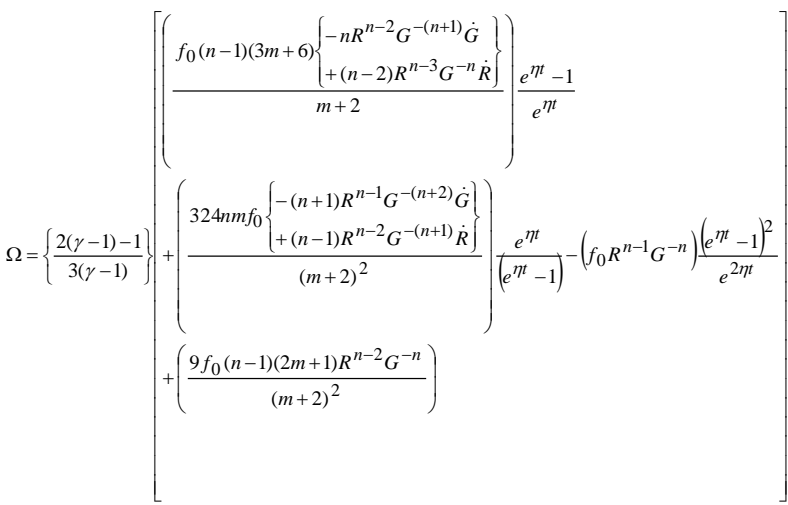

A behavior of an overall density parameter of dynamics of the bulk viscous string an cosmological model versus a time with the suitable choice of constants as shown inside the subsequent Fig. 12. Also this parameter gives us the overall density with three models of the universe.

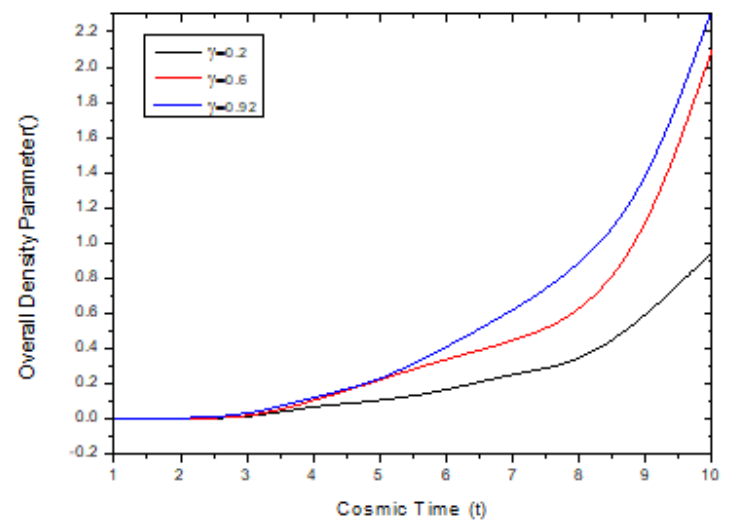

Fig. 12. Behavior of overall density parameter of dynamics of bulk viscous string cosmological model versus cosmic time with the appropriate choice of constants $m=2, n=3$ and $\eta=0.2$

\section{Conclusions}

In this circumstances with tension density equation of state (EoS), in the metric version of $f(R, G)$ gravity has been investigated. In the vigorous exploration of spatially unvaried and anisotropic locally rotationally symmetric Bianchi type-I cosmological model with dynamics of bulk viscous string provide among the framework of $f(R, G)$ theory of gravity at $t=0$, the model of the universe is constant and metric potentials ne'er approaches to vanishing worth. Also at $n=1$ or $n=0$ we get the reciprocal model of the universe. An exact solution of the field equations corresponds to special law of variation which provides singular model. We have evaluated some important cosmological physical and kinematical parameters for this model. The energy density, string tension density, energy density of particle, bulk viscosity of fluid and kinematical parameters $H, \theta$ and $\sigma^{2}$ all are infinite at this point but the volume scale factor vanishes. Also by jerk parameter it is observed that the model of universe shows regular bounce. 


\section{References}

[1] A. Pradhan, "Some Magnetized Bulk Viscous String Cosmological Models in Cylindrically Symmetric Inhomogeneous Universe with Variable $\Lambda$-Term", Commun. Theor. Phys. (Beijing,China), 51; pp. 367374, 2009.

[2] A. Czajka et al. "Bulk viscosity at extreme limits: from kinetic theory to strings", J. of High Energy Phys. 07, 145, 2019.

[3] B. K. Bishi and K. L, "Mahanta. Bianchi Type-V Bulk Viscous Cosmic String in Gravity with Time Varying Deceleration Parameter", Advances in High Energy Physics, volume 2015, 8 pages, 2015.

[4] C. P. Singh. "Some exact solutions of magnetized viscous model in string cosmology", PRAMANA Journal of Phys., vol. 83, no. 1, pp. 63-81, 2014.

[5] D. M. Gusu, "Bianchi Type-I Bulk Viscosity with a DE Cosmological Model", Advances in High Energy Physics, 10 pages, 2020.

[6] E. A. Hegazy, "Bulk viscous Bianchi type I cosmological model in Lyra geometry and in the general theory of relativity", Astrophys Space Sci. 365:119, 2020.

[7] H. Çağlar and S. Aygün, "Exact Solutions of Bulk Viscous with String Cloud Attached to Strange Quark Matter for Higher Dimensional FRW Universe in Lyra Geometry", AIP Conference Proceedings, 1722, $050001,2016$.

[8] K. S. Adhav et al. "String Cloud and Domain Walls with Quark Matter in n-Dimensional Kaluza-Klein Cosmological Model", Int J Theor Phys; 47:2002-2010, 2008.

[9] M. Sharif and S. Waheed, "Dynamics of Magnetized Bulk Viscous Strings in Brans-Dicke Gravity", Int. J. Mod. Phys. D, 21,1250055, 2012.

[10] M. K. Singh and S. Ram, "Dynamics of Anisotropic Bianchi Type-III Bulk Viscous String Model with Magnetic Field", Int. J. Theor. Phys; 2014.

[11] M. K. Singh et al. "Anisotropic Bianchi Type-II Viscous Fluid Models with Time-Dependent Gravitational and Cosmological Constants", International Journal of Physics, 1(4), pp. 77-83, 2013.

[12] M. S. Borkar and N. K. Ashtankar, "LRS Bianchi Type-II Cosmological Model with String Bulk Viscous Fluid and Magnetic Field in Barber's Second Self Creation Theory", AAM: Intern. J, Vol. 11, Issue 1, pp. 215228, 2016.

[13] M. Vijaya Santhi et al. "Bulk viscous string cosmological models in gravity", Canadian Journal of Physics, Vol. 96, No. 1, 2018.

[14] S. D. Katore et al. "Cosmology of string bulk viscosity in theory of gravitation", International Journal of Geometric Methods in Modern Physics, Vol. 15, No. 7, 1850116, 12 pages, 2018.

[15] S. P. Hatkar and S. D. Katore, "Anisotropic bulk viscous string cosmological models with heat flux", J. for Foundations and Applications of Phys, Vol. 5, No. 2, 2018.

[16] S. Ram et al. "Bulk viscous Bianchi-I cosmological model in) gravity theory", Phys. Astron. Int. J. 2(4):330-334, 2018.
[17] S. Ram and P. Kumari. "Bianchi types I and V bulk viscous fluid cosmological models in gravity theory", Cent. Eur. J. Phys. 12(10), 744754, 2014.

[18] T. Vidyasagar et al. "Bianchi type-VI0 bulk viscous string cosmological model in Brans-Dicke scalar-tensor theory of gravitation", Eur. Phys. J. Plus. 2, 129: 36, 2014.

[19] U. M. R. Velagapudi and N. Davuluri, "String cosmological models with bulk viscosity in Nordtvedt's general scalar-tensor theory of gravitation", Journal of Theoretical and Applied Physics, 2013.

[20] U. Y. Divya Prasanthi and Y. Aditya, "Kantowski-Sachs bulk viscous string cosmological model in theory of gravity", IOP Conf. Series: Journal of Physics: Conf. Series, 1344, 01204, 2019.

[21] V. U. M. Rao and D. Neelima, "Axially Symmetric Bulk Viscous String Cosmological Models in GR and Brans-Dicke Theory of Gravitation", Astronomy and Astrophysics, Vol. 2013, 5 pages, 2013.

[22] V. G. Mete et al. "Bianchi Type IX Magnetized Bulk Viscous String Cosmological Model in General Relativity", Theoretical Physics, Vol. 2, No. 1, 2017.

[23] A. Cruz-Dombriz and D. Saez-Gomez, "On the stability of the cosmological solutions in gravity", Class. Quantum Grav., 29, 245014, pp. 25, 2012.

[24] E Elizalde et al. " $\Lambda \mathrm{CDM}$ epoch reconstruction from and modified GaussBonnet gravities", Class. Quantum Grav, 27, 095007, pp. 13, 2010.

[25] M. De Laurentis et al. "Cosmological inflation in gravity", Physical Review D, 91, 083531, 2015.

[26] S. H. Shekh et al. "Thermodynamical aspects of relativistic hydrodynamics in gravity", International Journal of Geometric Methods in Modern Physics, IJGMMP-D, 19, 00459, 2020.

[27] S. D. Odintsov et al. "Dynamics of inflation and dark energy from gravity", Nuclear Physics B, 2018.

[28] S. H. Shekh. "Dynamical analysis with thermodynamic aspects of anisotropic dark energy bounce cosmological model in gravity", New Astronomy, 83, 101464, 2021.

[29] P. K. Sahoo et al. "Bianchi type string cosmological models in gravity", Eur. Phys. J. Plus., 131: 333, 2016.

[30] R. K. Mishra and H. Dua, "Bulk viscous string cosmological models in Saez-Ballester theory of gravity", Astrophys. Space. Sci., 364:195, 2019.

[31] S. D. Katore and A. Y. Shaikh, "Cosmological Model with Strange Quark Matter Attached to Cosmic String for Axially Symmetric Space-Time", Int J Theor Phys., 51:1881-1888, 2012.

[32] S. R. Bhoyar et al. "Accelerating Universe with Viscous Cosmic String in Quadratic Form of Teleparallel Gravity”, J. Sci. Res; 11(3), 249-262, 2019.

[33] K. P. Singh and J. Baro, "Bulk Viscous Fluid Bianchi Type-I String Cosmological Model with Negative Constant Deceleration Parameter", Advances in Mathematics: Scientific Journal, vol. 9, no. 7, pp. 49074916, 2020. 